\title{
Importancia de crear una Política Pública Abierta para el Logro de la Igualdad de Género
}

\author{
Importance of Create an Open Public Policy in Order to Achieve \\ Gender Equality
}

\begin{abstract}
Adria Velia González Beltrones, María Inés Aragón Salcido, Guadalupe Aleida Valenzuela Miranda, María Dolores Rocha Ontiveros, ${ }^{1}$ Ulises Torres Anaya ${ }^{2}$
\end{abstract}

\begin{abstract}
Sumario: Introducción. II Datos económicos en la gestación del dilema . III. IV. Presupuestos participativos con perspectiva de género. V Planteamiento del Problema. VI. Objetivo. VII Marco Teorico Conceptual VIII. Un poco de historia como insumo para crear o modificar una política pública abierta. IX. Desglose Jurídico. X.Pronóstico de consecuencias. XI. Metodología. XII Retos XIII. Bibliografía
\end{abstract}

\section{Resumen.}

El documento describe la importancia de crear una Política Pública Abierta, para alcanzar, mediante el presupuesto participativo con perspectiva de género,la igualdad entre hombres y mujeres en determinados rubros.

\section{Abstract.}

The paper describes the importance of create an Open Public Policy in order to achieve, through the participatory budget with gender perspective, equality between men and women in specific areas.

\section{Introducción}

La meta del Programa de Gasto Público es promover una Hacienda Pública ${ }^{3}$ responsable, eficaz, eficiente y transparente que promueva condiciones de bienestar entre los mexicanos.

\footnotetext{
${ }^{1}$ Docentes Investigadoras de la Universidad de Sonora

2 Estudiante de la licenciatura en Administración del Departamento de Contabilidad de la DCEA de la Universidad de Sonora URC

3 Comisión Económica para América Latina y el Caribe CEPAL. Benavente María Cristina, Valdés B Alejandra Políticas públicas para la igualdad de género. Un aporte a la autonomía de las mujeres. CEPAL Santiago de Chile,2014
} 
Para lograrlo, según se declara en México Evalúa ${ }^{4}$ - se desagrega el gasto público en unidades de análisis más cercanas conceptualmente y en la práctica al ciudadano, monitorea y evalúa la calidad del presupuesto y del gasto público ejercido, y analiza la normatividad y las instituciones que rigen el manejo de los recursos públicos con el fin de proponer mejoras en estos ámbitos, de acuerdo con las buenas prácticas internacionales.

En los últimos quince años, las finanzas públicas en México presentan una situación contrastante y por ello preocupante. Mientras que la recaudación de ingresos refleja la implementación de una política reactiva y en términos generales acertada, la política de gasto público se observa mucho menos estructurada. Consecuentemente, los ingresos obtenidos no han sido suficientes para compensar las presiones del gasto público y la deuda pública ha ido en aumento considerablemente. Las autoridades hacendarias justifican lo anterior, al señalar que son básicamente dos cuestiones las que han afectado seriamente los ingresos federales: la reducción en la producción de petróleo desde hace ya varios años y la caída de los precios del petróleo en el mercado internacional.

Ante esta perspectiva, las autoridades fiscales dirigieron su atención hacia los ingresos tributarios, incrementando las tasas y tarifas del Impuesto Sobre la Renta ISR primordialmente, así como las de los impuestos especiales, destacando en estos últimos, el de las gasolinas. La queja generalizada, de los ciudadanos afectados por tener que pagar más impuestos, se dejó escuchar a lo largo y ancho de nuestro país, y a nadie convenció el que las autoridades hayan respondido, que con estas medidas en la política de ingresos, se evitaba una factible crisis a nivel macroeconómico.

\section{Datos económicos en la gestación del dilema}

El verdadero problema entonces, no está en los ingresos, sino en la otra cara de la moneda: los egresos. Entre 2008 y 2015, el gasto presupuestal del gobierno federal aumentó más de 3.5 puntos porcentuales del PIB. En el presente sexenio ha

\footnotetext{
${ }^{4}$ México Evalúa consultado [on line] Disponible en:

http://mexicoevalua.org/2014/04/14/descifrando-la-caja-negra-del-gasto/
} 
aumentado cerca de dos puntos del $\mathrm{PIB}^{5}$. En cuanto a la composición del aumento también es preocupante ya que el gasto corriente programable ha aumentado 2.2 puntos del PIB desde 2008 y 0.8 puntos desde 2012. En contrapartida el gasto de capital programable ha aumentado 0.8 puntos desde el 2008 y 0.4 puntos desde 2012 . El resultado, es un aumento en el déficit público ${ }^{6}$, el cual, en 2015 llegó a poco más de 4.0\% del PIB. Y en consecuencia el aumento de la deuda pública en el periodo 20082015 en más de 14 puntos del PIB y casi 10 puntos desde el inicio de la presente administración.

En situaciones como la descrita, los expertos recomiendan una reducción del déficit, lo que implica un ajuste muy fuerte del gasto, y en todo caso, revisar y eficientar las políticas recaudatorias por el lado de los ingresos. En el mismo tenor se recomienda que el ajuste al gasto público se realice en el gasto corriente que genera pocos impactos y no en el gasto de inversión, para evitar inhibir el crecimiento económico. Lo anterior preserva la necesaria, deseable y correcta evaluación que las autoridades gubernamentales deben hacer de los efectos del gasto público, para gastar cada peso, en aquellas actividades que beneficie al mayor número de personas atendiendo así a los principios del gasto público.

Ahora bien, en relación a una de las tareas pendientes del estado mexicano como lo es el logro de la igualdad de género, las políticas públicas que inciden positivamente en ello son "una herramienta fundamental para impulsar las transformaciones hacia mayores niveles de justicia, además de expresar la decisión política de los gobiernos de avanzar en la solución de los problemas de desigualdad que afectan a las mujeres."

Al decir de los expertos "los distintos grados de avance en relación con la igualdad de género en los países de la región de América Latina y del Caribe muestran que todavía

5 SHCP,Secretaría de Hacienda y Crédito Público,[online] Disponible en: http://finanzaspublicas.hacienda.gob.mx/es/Finanzas_Publicas/Informes_al_Congreso_ de_la_Union Consultado 20/03/2016

6 Expansión datosmacro [online]Disponible en:http://www.datosmacro.com/deficit , Consultado 20/03/2016

${ }^{7}$ Comisión Económica para América Latina y el Caribe, CEPAL Políticas Públicas para la Igualdad de Género Un Aporte a la Autonomía de las Mujeres ,Benavente R. María Cristina, Valdés B. Alejandra,Santiago de Chile, 2014,pp6 
hay un largo camino por recorrer, especialmente en cuanto al fortalecimiento de las capacidades estatales para la puesta en marcha efectiva de políticas públicas orientadas a disminuir las brechas de género." 8 pues si bien las políticas de género "se pueden analizar como buenas prácticas, es importante desarrollar un debate experto, a fin de elaborar una matriz de análisis para la identificación y sistematización de políticas, que considera las distintas fases del ciclo de las políticas públicas, a fin de describir y analizar políticas que puedan ser calificadas como justas para la igualdad de género." En esta línea como un ejemplo de análisis de políticas seleccionadas previamente, ---en la medida en que responden a nuevos derechos a reconocer o a situaciones preexistentes de extrema desigualdad, lo son: el reconocimiento del trabajo no remunerado realizado en el hogar y el reconocimiento de la existencia de dimensiones críticas y extremas de desigualdad, como la violencia intrafamiliar.

En este sentido y acorde a los planteamientos de la Comisión Económica para América Latina y el Caribe CEPAL, los profundos desequilibrios económicos, sociales y ambientales han motivado la búsqueda de respuestas por parte de la comunidad internacional, a través de esfuerzos que han madurado por más de dos decenios. La más amplia y ambiciosa es la Agenda 2030 para el Desarrollo Sostenible y los 17 Objetivos de Desarrollo Sostenible (ODS), que los 193 países representados en la Asamblea General de las Naciones Unidas aprobaron en septiembre de 2015. Estos compromisos reconocen la igualdad y la sostenibilidad como los principios rectores, compartidos y universales, en los que se debe basar una nueva batería de estrategias y políticas globales, regionales y nacionales. La masiva participación de la comunidad internacional en su formulación y aprobación les confiere una legitimidad sin

${ }^{8}$ Pues como bien subraya la CEPAL, el identificar políticas públicas de igualdad de género de carácter redistributivo, de reconocimiento y de representación es un esfuerzo por relevar las iniciativas de los Estados que se orientan a enfrentar injusticias y mejorar la situación de las mujeres en distintas áreas de desarrollo, de manera de hacer posible su autonomía y la garantía de sus derechos humanos en contextos de plena igualdad. Comisión Económica para América Latina y el Caribe, CEPAL Políticas Públicas para ...op.cit,pp7

${ }^{9}$ Comisión Económica para América Latina y el Caribe, CEPAL Políticas Públicas para... ibid.pp7 
precedentes y refuerza el compromiso político de los países de implementarlos eficaz y eficientemente, ajustándolos en la medida en que lo demanden los grandes cambios en curso.

En este tenor, resulta altamente alarmante el saber que América Latina y el Caribe enfrenta los desafíos de la Agenda 2030 para el Desarrollo Sostenible con una tasa de crecimiento de largo plazo inferior a la de otras regiones del mundo en desarrollo y una persistente vulnerabilidad externa. ${ }^{10}$

Las anteriores características condicionan el que acontecimientos como el virtual estancamiento de la economía mundial, la volatilidad financiera, los paraísos fiscales, las migraciones masivas, las caídas repentinas de los precios de los productos básicos entre otras, afectan a la región en su conjunto y a sus subregiones. Desde hace un Iustro, la mayor parte de los países han experimentado una desaceleración del ritmo de crecimiento económico, que se ha convertido, en algunos casos, en una contracción. El componente de la demanda agregada más afectado por la desaceleración es la inversión, lo que tiene implicaciones negativas en la productividad y la competitividad. Al mismo tiempo, se redujo el espacio fiscal en casi todos los países. La combinación de estos factores indica que la región deberá cambiar su estilo de desarrollo en condiciones menos favorables que en la década pasada, con menos margen de maniobra y una pérdida relativa de capacidades tecnológicas. ${ }^{11}$

${ }^{10}$ Su dinámica de crecimiento sufrió caídas derivadas de los choques económicos causados por la crisis de la deuda externa en los años ochenta, las crisis mexicana y asiática, rusa y brasileña en los noventa, y la crisis argentina, seguida de la crisis financiera mundial, en la década de 2000. Peor aún, en los períodos de auge no ha tenido un desempeño destacado en la comparación internacional: su tasa de crecimiento en el período 2003-2007, la mejor de las últimas tres décadas, fue más baja que la de otras regiones del mundo en desarrollo. Asimismo, persiste su vulnerabilidad externa real y financiera; debido a los patrones de especialización predominantes, sus países siguen siendo sensibles a choques en los términos de intercambio y la demanda externa, en algunos casos han aumentado sus pasivos externos brutos y dependen en mayor medida del ingreso de capitales internacionales. Horizontes 2030 La igualdad en el centro del desarrollo sostenible, Comisión Económica para América Latina y el Caribe CEPAL, Trigésimo Sexto período de sesiones, Ed. CEPAL Ciudad de México, 23 a 27 de mayo de 2016,pp.9

${ }^{11}$ CEPAL,op.cit pp.10 
Por su parte, en la misma línea, la $\mathrm{OCDE}^{12}$ apunta entre otros aspectos, que México sigue teniendo fuertes desigualdades en el ingreso, deficiencias en materia de igualdad de género y severas disparidades regionales.....por lo tanto la reducción de la desigualdad y la inclusión deben tener prioridad en la agenda de reformas. Esto es importante por razones de bienestar, pero también porque las sociedades más incluyentes tienden a lograr un mejor desempeño.

\section{Presupuestos participativos con perspectiva de género}

En este contexto en relación a las políticas públicas orientadas a disminuir las brechas de género tenemos como herramienta principal a los presupuestos participativos con perspectiva de género, ya que a partir del reconocimiento de que no existen presupuestos públicos neutrales en materia de género y de que la invisibilidad de las cuestiones de género en estos podía agravar las desigualdades existentes, se constata la necesidad de contar con presupuestos con perspectiva de género.

Si se analiza un presupuesto de egresos confeccionado en forma tradicional, con orientación programática, o por programas, presentado por alguna instancia gubernamental en nuestro país se puede constatar la insuficiencia de los recursos públicos destinados a atender y solucionar la problemática de género que afecta directamente a las mujeres, en cuestiones como la salud reproductiva, la mortalidad materna, la falta de acceso a recursos económicos y la violencia, entre otras, de manera tal que se hace patente la necesidad de etiquetar gastos en esos ámbitos y confeccionar un presupuesto público con perspectiva de género ${ }^{13}$.

${ }^{12}$ Organización para la Cooperación y el Desarrollo Económicos OCDE, Serie Mejores Políticas, México Mejores Políticas para un Desarrollo Incluyente, Ed. OCDE, Septiembre 2012,pp7

${ }^{13}$ Como ejemplo de ello tenemos el caso de la transición en nuestro país a nivel federal de un presupuesto tradicional a un presupuesto participativo con perspectiva de género ya que "durante el proceso de confección de ese presupuesto se identificó la necesidad de crear un marco jurídico y normativo que hiciera sostenible, con miras a fomentar la transversalidad, la asignación y ejecución de recursos públicos destinados a las mujeres y a la igualdad de género. Asimismo, se demostró la existencia de una cultura institucional y organizacional androcéntrica predominante que obstaculizaba la aplicación de las disposiciones legales y normativas. Comisión Económica para América Latina y el Caribe, CEPAL Políticas ....ibid.,pp.97 
Ahora bien, la convocatoria del pasado primero de marzo del presente año, suscrita por el Sistema Nacional de Transparencia, Acceso a la Información Pública y Protección de Datos Personales, a través de la Comisión de Gobierno Abierto y Transparencia Proactiva; ${ }^{14}$ justifica en forma expresa su expedición, en su parte correspondiente, con el siguiente texto: "Continuar avanzando hacia la consolidación de un Estado Abierto en México, considerando como punto de partida la implementación de ejercicios de Gobierno Abierto a nivel local, basados en la transparencia y la participación ciudadana en un ambiente de rendición de cuentas e innovación (cívica y tecnológica), con la finalidad primordial de dar atención y eventual solución colaborativa, a problemas públicos “

En ese sentido y en aras de coadyuvar en el fomento de lógicas colaborativas entre ciudadanos y autoridades, presentamos la siguiente problemática, que atañe en forma específica a una parte (mujeres)de la población de nuestro estado y de la de sus municipios, y en su conjunto al total de su población en los aspectos político, social y económico; para enseguida justificar la urgencia mas allá del diseño de una política pública abierta, la urgencia de la voluntad política para su ejecución y permanente evaluación en relación a los propósitos trazados. .

\section{Planteamiento del problema}

En nuestro país, mientras la mayor parte de sus entidades federativas y sus municipios no elaboren sus presupuestos de egresos en forma participativa y con perspectiva de género, el presupuesto continuará actuando como instrumento reproductor de las desigualdades de género y contribuirá a que persista $y$, probablemente, a que se agrave la situación desfavorable de las mujeres en la sociedad mexicana.

\section{Diagnóstico de la Situación en Sonora}

\footnotetext{
${ }^{14}$ con fundamento en los artículos 2, fracciones VI y VII, 27, 28, 29, 30, 31 fracciones III y IX y 59 de la Ley General de Transparencia y Acceso a la Información Pública; así como 27, fracción VII y 34 de los Lineamientos para la Organización, Coordinación y Funcionamiento de las Instancias de los Integrantes del Sistema Nacional de Transparencia, Acceso a la Información Pública y Protección de Datos Personales, y como parte de los ejercicios de promoción de lógicas colaborativas en los Estados del país para impulsar el gobierno abierto a nivel local.
} 
La Ley de Participación Ciudadana del Estado de Sonora establece dentro de los instrumentos de participación ciudadana, el presupuesto participativo ${ }^{15}$, con efectos vinculantes para el Ejecutivo del Estado y de los Ayuntamientos de los municipios, e incorporar además, la perspectiva de género en los programas y presupuestos, en concordancia con la Ley para la Igualdad entre Mujeres y Hombres del Estado de Sonora, en la cual se definen entre otros,los conceptos de igualdad sustantiva, equidad de género, perspectiva de género y la transversalidad, que ya deberían estarse aplicando en los programas y presupuestos de las administraciones públicas estatal y municipales, respectivamente. Sin embargo, de la revisión de los Boletines Oficiales y de los informes trimestrales del gobierno del Estado y de los Municipios al Instituto Superior de Auditoria y Fiscalización del Congreso del Estado, que aparecen en los portales de transparencia, encontramos que únicamente los municipios de Bacoachi por Acuerdo de Cabildo y el de Empalme, en forma transversal, aplican desde 2013 dicho instrumento de participación ciudadana incorporando la perspectiva de género. A partir del 2016 se sumaron 27 municipios más a la elaboración de sus presupuestos de egresos con perspectiva de género,24 no la contemplan y de 19 no se cuenta con información. Por lo que hace a la "participación ciudadana" --según el dispositivo de la Ley de la materia, todos los municipios pretenden dan por cumplida la "participación ciudadana", al considerar que, durante las campañas electorales recogieron las mas sentidas necesidades de sus comunidades las cuales se "priorizan" en sus planes de desarrollo y en sus presupuestos de egresos respectivos.

A nivel nacional, la Federación, la recién denominada Ciudad de México, Oaxaca y aproximadamente una tercera parte de las demás entidades federativas cuentan ya con presupuestos participativos con perspectiva de género. Por ello, consideramos que este problema debe ser prioritario en las agendas públicas para generar no solo sus posibles soluciones por medio de esquemas participativos y de co creación ciudadana sino además, encontrar la formula efectiva para su participación permanente en el diseño, e implementación no solo de la política pública abierta efectiva para el

\footnotetext{
${ }^{15}$ como una metodología a observar tanto por el gobierno del estado como por los 72 municipios de la entidad. Esta ley entró en vigor en julio de 2011; por lo tanto, los presupuestos desde el año 2012 y hasta la fecha debieron haber sido formulados con base en dicho ordenamiento;
} 
propósito delineado sino también en el uso de otras herramientas útiles al logro ulterior de sus objetivos como lo es el presupuesto participativo con perspectiva de género.

\section{Marco Teorico Conceptual}

Acorde a Insulza José (2005:9) El Gobierno abierto es una política pública que agrupa los conceptos de transparencia, participación y colaboración de los ciudadanos en las políticas públicas en donde la información y datos gubernamentales juegan un rol esencial.

Lee and Kwak (2011) sugieren que existiría un modelo de implementación del open government que pasaría por cuatro etapas: 1) mayor nivel de transparencia en los datos, u open data; 2) mejora en los niveles de participación abierta al ciudadano; 3) mejora en los niveles de colaboración abierta a la sociedad; 4) alcance de la implicación ciudadana permanente. De acuerdo a dicho patron, -si se situara- a los diferentes modelos de open government actualmente en marcha en las diferentes fases. Seguramente, ninguno pasaría de la fase dos.

Asimismo,De Ferrari and Fajardo (2005:355) señalan que Solo podremos hablar de un verdadero gobierno abierto cuando exista un marco regulatorio que garantice el derecho de acceso a la información, una política de combate contra la corrupción, y regulaciones claras en materia de datos abiertos, que permitan el libre acceso a la información clave para que los ciudadanos tomen decisiones para su vida cotidiana y puedan ejercer el control social, con definiciones claras en materia de participación ciudadana. Esto es un paso fundamental para profundizar nuestras democracias.

De acuerdo con Álvaro Ramírez-Alujas (2011:103), el concepto de gobierno abierto actualmente trasciende por mucho estas definiciones, puesto que "fluye desde la convicción de repensar la administración pública, de transformar la sociedad y contribuir a desarrollar democracias más sanas, de pasar de las jerarquías a las redes, de asumir compromisos transversales y generar, en conjunto con otros actores sociales y económicos, valor público". Esta acepción del término parece ser la más ambiciosa, pues supone un cambio paradigmático a través de la ejecución de un modelo en donde la participación sustantiva de la ciudadanía forme parte de la toma de decisiones en los asuntos públicos. 
Las políticas públicas en nuestros dias, no son solo necesarias sino también indispensables.Con la intención de avanzar por este camino, se busca la construcción de un proyecto social de alcances mayores que atienda las condiciones que prevé todo esquema de actuación comprometida de Gobierno Abierto: Transparencia, Participación y Colaboración.

Señalan los expertos que el Gobierno Abierto tiene un amplio campo de acción tanto al interior de la Administración Pública como al exterior de la misma, actualmente de una u otra forma se trabaja en conjunto con la sociedad civil en la co-creación de políticas públicas y avances concretos al respecto, es un campo bastante amplio para trabajar y de evolución vertiginosa gracias a la también veloz evolución de las TIC, ejemplo de ello el Gobierno Electrónico, promoviendo aún más la transparencia y rendición de cuentas, pero asociadas a mecanismos de apertura, datos y mecanismos efectivos de participación ciudadana, desarrollando herramientas con elementos que aumenten la participación de los usuarios, información reutilizable, información en formatos de datos abiertos.

Si bien la intención es, que la información relevante y de mayor utilidad o uso frecuente pueda ser aprovechada por los ciudadanos, para realizar con ella, propuestas de trabajo, o de proyectos como solución a las diversas problemáticas de su entorno, que logren incidir en la toma de decisiones de los gobernantes, lo cierto es que en este aspecto en nuestro pais y en nuestros estados y sus municipios aun falta camino por recorrer para la obtención de resultados benéficos para la sociedad, que se reflejen en la mejora de los servicios públicos.

\section{Un poco de historia como insumo para crear o modificar una política pública}

Desde finales de la Segunda Guerra Mundial SGM se produjo la adopción generalizada del Estado del Bienestar, prácticamente por todos los países del Orbe. México como integrante de la Organización para la Cooperación y el Desarrollo Económicos ${ }^{16}$ OCDE, cuenta con - políticas de financiamiento del desarrollo ${ }^{17}$, cuyos

\footnotetext{
${ }^{16}$ Fundada en 1961, la Organización para la Cooperación y el Desarrollo Económicos (OCDE) agrupa a 34 países miembros y su misión es promover políticas que mejoren el bienestar económico y social de las personas alrededor del mundo.

17 Mediante Decreto publicado en el Diario Oficial de la Federación el 20 de mayo de 2013 se aprobó el Plan Nacional de Desarrollo 2013-2018, el cual contiene los objetivos, estrategias, indicadores y metas que regirán la actuación del Gobierno Federal durante la.... administración(2012-2018) . El Plan Nacional de Desarrollo 20132018 prevé como estrategia general elevar la productividad para llevar a México a su
} 
rasgos a destacar es el importante crecimiento experimentado en el gasto público, tendiente a la consolidación del Estado del Bienestar ${ }^{18}$ mediante un esfuerzo inversor

máximo potencial, por lo que se orienta la actuación gubernamental en torno a cinco metas nacionales: México en Paz, México Incluyente, México con Educación de Calidad, México Próspero y México con Responsabilidad Global, incluyendo además tres estrategias transversales: Democratizar la Productividad, Gobierno Cercano y Moderno y Perspectiva de Género. La meta nacional México Próspero, tiene por objeto, entre otros, mantener la estabilidad macroeconómica del país; democratizar el acceso al financiamiento de proyectos con potencial de crecimiento; fortalecer los ingresos del sector público, y promover un ejercicio eficiente de los recursos presupuestarios disponibles.

de Financiamiento del Desarrollo 2013-2018.

${ }^{18}$ El estado de bienestar en México se constituye por un complejo de sistemas de seguridad social formado por el Instituto Mexicano del Seguro Social (IMSS), el Instituto de Seguridad Social al Servicios de los Trabajadores del Estado (ISSSTE), los servicios de seguridad social del Ejército, la Marina, Pemex, la Comisión Federal de Electricidad, las universidades públicas, la banca de desarrollo y los que tienen establecidos los gobiernos estatales como es el caso del ISSSTECH,ISSSTESON en Chihuahua y en Sonora respectivamente. Mediante estos sistemas se calcula atender aproximadamente al 65 por ciento de la población del país (72.3 millones de derecho habientes), el resto se encuentra afiliado casi en su totalidad al Seguro Popular, los servicios de la Secretaría de Salud y el Programa IMSS Oportunidades, que de acuerdo a cifras oficiales supera los 50 millones de personas. No obstante, de acuerdo con los últimos informes del INEGI, sólo el 36.4 por ciento (alrededor de 40 millones de personas) es usuaria de los servicios de salud de los institutos de seguridad social, en tanto que en los servicios de protección social (SSA, Seguro Popular, IMSS Oportunidades) son atendidos el 35.9 por ciento (39.5 millones de personas) y el restante 27.6 por ciento (poco más de 30 millones de personas) acude a servicios médicos privados. Lo que indica que aun contando con cobertura pública de salud, poco más de la cuarta parte de los mexicanos paga por su cuenta servicios médicos de particulares.Cabe señalar además, que la población derechohabiente a los sistemas de seguridad social (quienes realizan aportaciones acordes a su salario), tiene acceso a servicios de salud, derecho a una pensión de retiro, previsiones por discapacidad, cesantía o muerte, y prestaciones diversas como vivienda, préstamos, actividades culturales, entre otras. En tanto que los afiliados a los servicios de protección social (no contributiva) sólo cuentan con acceso a servicios de salud que según estudio del organismo México Evalúa, estos son cinco veces menos eficientes que los prestados por los institutos de seguridad social. En cuanto a las pensiones para la población no derechohabiente, hasta ahora se habían llevado esfuerzos aislados por otorgar una pensión no contributiva acorde con el límite mínimo de la línea de pobreza, por parte del gobierno federal a través del programa 70 y más, y de algunas entidades federativas a partir de los 65 años, entre las que se encuentran el Distrito Federal y Chiapas. Es a partir de 2015 que se establece la pensión universal a adultos mayores de 65 años, de acuerdo con las bases constitucionales recientemente aprobadas y con la promulgación de la Ley de Pensión Universal. 
aceptable. Así en el Programa Nacional de Financiamiento del Desarrollo 2013-18 ${ }^{19}$ PRONAFIDE, se contemplan tres Estrategias Transversales establecidas en el Plan Nacional de Desarrollo 2013-2018 .... las que se ven reflejadas en los diversos objetivos de dicho Programa Nacional de Financiamiento del Desarrollo 20132018:Democratizar la Productividad ${ }^{20}$, Gobierno Cercano y Moderno ${ }^{21}$ y Perspectiva de

\footnotetext{
${ }^{19}$ Ver Tercera Sección, Diario Oficial de la Federación del Lunes 16 de diciembre de 2013

${ }^{20}$ Las líneas de acción para Democratizar la Productividad, además de incorporarse de manera especifica en cada uno de los objetivos del PRONAFIDE se reflejan de manera general en las siguientes estrategias:
}

Objetivo 1 Ejercer una política fiscal responsable que fomente el desarrollo económico: Proteger las finanzas públicas ante riesgos del entorno macroeconómico. Fortalecer la estructura de la deuda, promover el desarrollo del mercado, consolidar el acceso a las fuentes de financiamiento y reducir los costos del mismo. Fortalecer el marco institucional relevante para orientar el gasto, los programas y políticas públicas a elevar y democratizar la productividad.

Objetivo 2 Contar con un sistema hacendario que genere mas recursos, sea simple, progresivo y que fomente la formalidad: Garantizar la implementación adecuada de las medidas de la Reforma Hacendaria diseñadas para incrementar la capacidad financiera del Estado Mexicano. Adoptar plenamente las medidas de la Reforma Hacendaria orientadas a simplificar las disposiciones fiscales y facilitar la incorporación de un mayor número de contribuyentes al padrón fiscal. Implementar las medidas de la Reforma Hacendaria para fomentar a la formalidad.

Objetivo 5 Fomentar la inclusión, educación, competencia y transparencia de los sistemas financiero, asegurador y de pensiones para incrementar su penetración y cobertura, a la vez que mantengan su solidez $y$ seguridad: Ampliar la cobertura del sistema financiero, en particular hacia sectores excluidos, con perspectiva de género y empresas con potencial productivo. Promover el financiamiento en mejores condiciones para fomentar un mayor bienestar y desarrollo económico, dentro del marco de solidez y prudencia del sistema financiero. Implementar el Seguro de Desempleo y la Pensión Universal.

Objetivo 6 Ampliar el crédito de la Banca de Desarrollo facilitando el acceso a servicios financieros en sectores estratégicos, con una mayor participación del sector privado: Impulsar un mayor otorgamiento de crédito con especial énfasis en áreas prioritarias para el desarrollo 


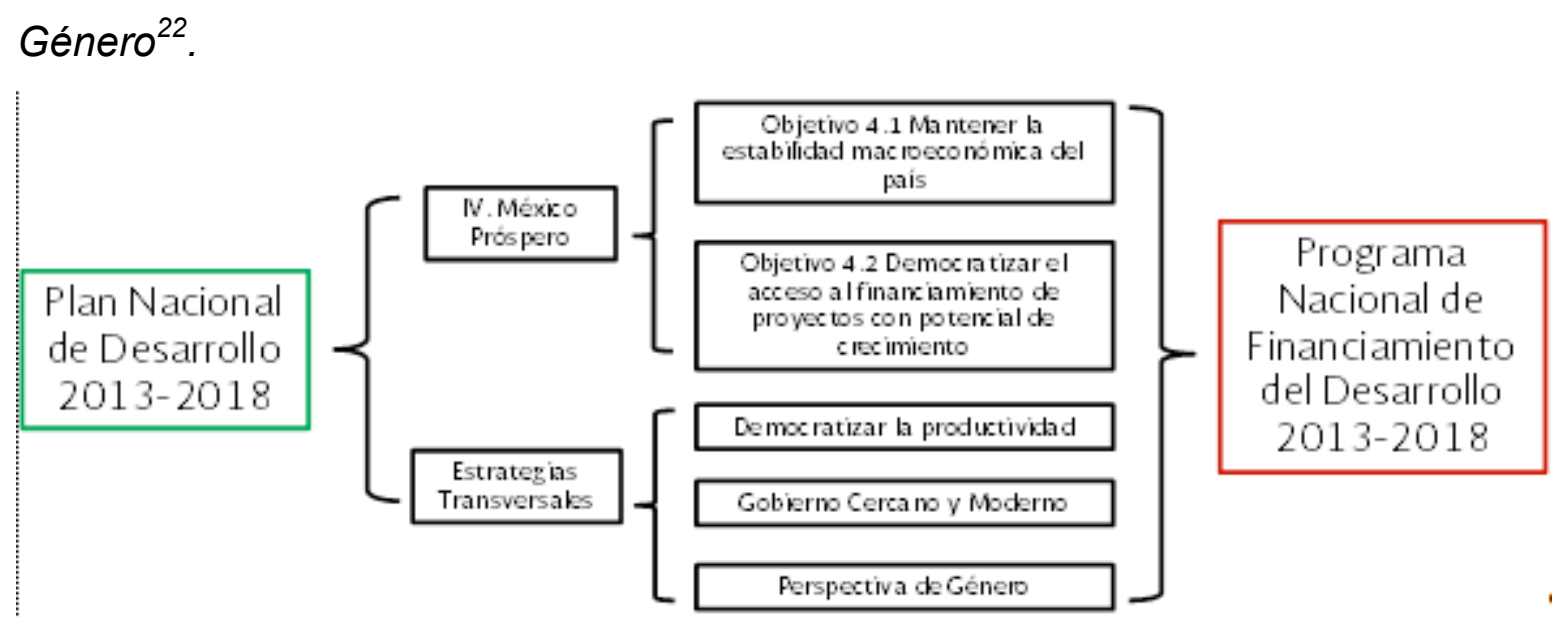

Fuente: DOF Tercera Sección, Diario Oficial de la Federación del Lunes 16 de diciembre de 2013

En relación a la evolución del gasto público nacional en su conjunto respecto a la normatividad contemplada en el PRONAFIDE 2013-2018 ${ }^{23}$-- y la realidad que se pretende abatir con su aprobación, aún existen diferencias no obstante que éstas vayan progresivamente descendiendo. Esto es así, porque funciones tan importantes desde la perspectiva del estado de bienestar- como la salud, educación, protección

nacional. Fortalecer los programas de garantías para aumentar los préstamos y mejorar sus condiciones. Promover esquemas que permitan mayor Inclusión Financiera en la población que enfrente limitantes para acceder al crédito y a otros servicios financieros

${ }^{21}$ Las líneas de acción transversales correspondientes al Programa para un Gobierno Cercano y Moderno, se atenderán mediante bases de colaboración suscritas entre la Secretaría de Hacienda y Crédito Público y la Secretaría de la Función Pública.

${ }^{22}$ Las siguientes estrategias del PRONAFIDE coadyuvan a la implementación de las líneas de acción transversales correspondientes a la Estrategia Transversal de Perspectiva de Género:

Objetivo 5 Fomentar la inclusión, educación, competencia y transparencia de los sistemas............

Objetivo 6 Ampliar el crédito de la Banca de Desarrollo facilitando el acceso a servicios................

${ }^{23}$ Conforme a la cual se presenta los objetivos, estrategias y líneas de acción que el Gobierno de la República implementará en los sectores hacendario y financiero para asegurar la disponibilidad de los recursos fiscales y financieros necesarios para el desarrollo de México. En específico, el programa se enmarca dentro de la Meta Nacional México Próspero del PND con sus objetivos y estrategias transversales. 
social y vivienda ${ }^{24}$ muestran serias deficiencias en la cobertura de esos gastos en nuestro país, situación ésta que podría estar apuntando la dirección a seguir en el futuro respecto a su ejecución ${ }^{25}$.

${ }^{24}$ INFONAVIT Instituto del Fondo Nacional para la Vivienda de los Trabajadores, Plan de Labores y Financiamientos 2016. Fundado en 1972, el INFONAVIT ha apoyado a más de 8 millones de trabajadores y sus familias para incrementar su patrimonio y les ha dado la posibilidad de acceder a una mayor calidad de vida. El valor social que el INFONAVIT aporta a las familias mexicanas es palpable, ya que otorga más de $70 \%$ de la originación de créditos hipotecarios en México; esto lo convierte en el principal actor del mercado y en la institución financiera que atiende al mayor número de trabajadores activos del país, más de 17 millones. Prácticamente uno de cada cuatro mexicanos habita una vivienda financiada por el Infonavit. Sin embargo En entrevista después de encabezar la séptima reunión nacional de los trabajadores del INFONAVIT, su director Penchyna Grub, admitió que hay un rezago en la atención de la demanda de vivienda, pues en 44 años sólo han otorgado 9 millones de créditos, cuando la demanda es de alrededor de 20 millones. El INFONAVIT se ha sumado a una estrategia interinstitucional, encabezada por la Secretaría de Desarrollo Agrario, Territorial y Urbano (Sedatu), con la finalidad de facilitar la ampliación de las viviendas. Esta iniciativa busca construir un cuarto adicional en aquellas viviendas financiadas por el INFONAVIT que actualmente cuentan con una sola habitación y que por cambios en las necesidades de las familias requieran más espacio. Los principales beneficios son el combate al hacinamiento, la mitigación de la violencia intrafamiliar y, por ende, una mejor calidad de vida para los derechohabientes y sus familias. El análisis detallado de las necesidades de los trabajadores nos ha permitido entender que muchos de ellos requieren mudarse para aprovechar oportunidades laborales. Por ello, el Instituto se encuentra diseñando una solución que permita la movilidad hipotecaria, esto es, que posibilite a los acreditados del INFONAVIT intercambiar su vivienda por otra que cumpla mejor con sus necesidades. El Instituto cuenta con el $22 \%$ de los recursos registrados en las Administradores de Fondos para el Retiro (Afores), convirtiéndose en uno de los actores más relevantes en cuanto a la administración del ahorro de los trabajadores. El segundo mandato del Instituto, representa en la actualidad un área de oportunidad para generar mayor valor económico a los trabajadores y a sus familias. Consultado el 26/03/16 [on line] Disponible en:

http://portal.infonavit.org.mx/wps/wcm/connect/5a5a868c-f72e-4efb-be9f-

167a557ce29a/Plan_de_Labores_y_Financiamientos_2016.pdf?MOD=AJPERES\&CVI $\mathrm{D}=\mathrm{lb} 2 \mathrm{yf} 82$

${ }^{25}$ En México, el crecimiento económico en los últimos 20 años no ha sido satisfactorio. El porcentaje de la población en situación de pobreza se ha mantenido prácticamente sin cambios en las últimas dos décadas. En 2012, el 45 por ciento de la población se encontraba en situación de pobreza, mientras que el crecimiento anual promedio del PIB per cápita ha sido de sólo 1.2 por ciento en términos reales entre 1990 y 2011 . Si bien el crecimiento económico no es un fin en sí mismo, es una de las principales herramientas para combatir la pobreza y mejorar las condiciones de vida de la población. A nivel internacional, existe una fuerte correlación entre el crecimiento económico y la reducción de la pobreza, así como con el acceso a servicios relacionados con la salud, la nutrición y la educación. 
En el conjunto de los países del orbe, esta preocupación del Estado por la prestación de servicios sociales inicia a mediados de la década de los cuarenta del siglo pasado, aunque su desarrollo no haya sido uniforme en todos los países, dado los acontecimientos sociales, políticos y económicos particulares. Los avances de la macroeconomía, el clima social y político posterior a la Segunda Guerra Mundial SGM y los cambios en muchos de los estudios sobre la realidad social, establecieron las bases para legitimar la participación del Estado en la regulación de ciertos aspectos tanto económicos como sociales, pasando del estado-gendarme al estadointervencionista, hacia el estado del bienestar ${ }^{26}$ hasta llegar en el presente Siglo XXI en ciertas democracias a la adopción plena de modelos de gobiernos abiertos con políticas publicas abiertas. En el artículo 31 fracción IV de nuestra Carta Magna se consagra el principio de justicia en la imposición de Adam Smith, desdoblado en los de proporcionalidad y equidad al establecer el deber de todos, de contribuir al gasto público de manera proporcional y equitativa; y si bien para la mayoría de la doctrina, hasta antes de la Reforma Constitucional de junio de 2011 en materia de derechos humanos, esta obligación había sido considerada como un mero postulado, en los últimos años, dada la importancia en torno a que los recursos tributarios deben destinarse a satisfacer las necesidades colectivas por la administración pública directa, los diferentes sectores de la sociedad, con análisis mas metódicos al respecto exigen con mayor contundencia a los gobiernos federal y de las entidades federativas y sus municipios, la demanda de solución a los problemas que los afectan. ${ }^{27}$

En este contexto cabe recordar que en toda crisis económica recurrente se presentará el natural debate sobre las dimensiones y prioridades del gasto público. Pero, ¿que es

26 cuyo fundamento estriba en la intervención y en el principio de solidaridad, efectuándose el reparto de la riqueza a través de los sistemas de protección social, ${ }^{27}$ Ejemplos lo son las demandas magisteriales en voz de la Coordinadora Nacional de $\begin{array}{llll}\text { Trabajadores de la } & \text { Educación } & \text { CNTE, }\end{array}$ http://www.excelsior.com.mx/nacional/2016/07/27/1107360, demandas de los empresarios Coparmex demanda al gobierno por omisiones ante actos de la CNTE, del senado por medio de su presidente Roberto Gil Zuarth, demandó al gobierno federal poner fin al chantaje y al boicot de la CNTE a la educación, los empresarios Coparmex demanda al gobierno por omisiones ante actos de la CNTE los empresarios 
gasto público? Siguiendo al jurisconsulto Emilio Margain Manautou, y amplificando el concepto ${ }^{28}$ son las erogaciones que realiza el Estado a través : de la administración activa del poder ejecutivo, de los otros dos poderes que integran la Unión, de los órganos autónomos, de las entidades federativas y de los Municipios, destinadas a satisfacer las funciones de sus entidades, en los términos previstos en el presupuesto de egresos $^{29}$ cuya finalidad es dar cumplimiento al Artículo 25 Constitucional que en su primer párrafo establece que

Corresponde al Estado la rectoría del desarrollo nacional para garantizar que éste sea integral y sustentable, que fortalezca la Soberanía de la Nación y su régimen democrático y que, mediante la competitividad, el fomento del crecimiento económico y el empleo y una más justa distribución del ingreso y la riqueza, permita el pleno ejercicio de la libertad y la dignidad de los individuos, grupos y clases sociales, cuya seguridad protege esta Constitución. La competitividad se entenderá como el conjunto de condiciones necesarias para generar un mayor crecimiento económico, promoviendo la inversión y la generación de empleo. ${ }^{30}$

En este sentido,el marco jurídico nacional para que dichos postulados se cumplan: por un lado a través del diseño del Plan Nacional de Desarrollo en el cual se plasman las prioridades de la población y fines del Estado; de la Ley Federal de Presupuesto y Responsabilidad Hacendaria, así como lo establecido en el Presupuesto de Egresos de la Federación y en los ordenamientos jurídicos locales homólogos a los precitados. ${ }^{31}$

${ }^{28}$ Margain Manautou Emilio,Introducción al Estudio del Derecho Tributario,Ed.Porrúa, México, 2014, pp.140

${ }^{29}$ Para el Ejercicio Fiscal 2016, el Ejecutivo Federal somete a la consideración de la Cámara de Diputados del $\mathrm{H}$. Congreso de la Unión, un gasto neto total equivalente a $4,746.9$ mil millones de pesos, $1.9 \%$ menos en términos reales que en el presupuesto aprobado de 2015.

Del total, $75 \%$ corresponde a gasto programable, menor en $5.8 \%$ en términos reales al aprobado de 2015, y 25\% restante corresponde a erogaciones de naturaleza no programable, que se estiman crezcan en $12.2 \%$ respecto al monto aprobado para el Ejercicio Fiscal 2015, una vez descontado el efecto del incremento en los precios.

30 Artículo 25 Primer párrafo, Constitución Política de los Estados Unidos Mexicanos CPEUM 2016

${ }^{31}$ En el caso del estado de Sonora : Ley de Planeación del Estado de Sonora (Reforma del 23/Dic/1991);Ley de Presupuesto de Egresos, Contabilidad Gubernamental y Gasto 
En cuanto al marco jurídico federal que respalda la política pública en relación a la igualdad de género, descansa en un conjunto de normas, que se detallan a continuación:

\section{Desglose Jurídico}

1. La Constitución Política de los Estados Unidos Mexicanos, que en su artículo primero señala que todas las personas gozarán de los derechos humanos reconocidos en ella y en los tratados internacionales de los que el Estado mexicano sea parte.

2. La Ley Federal de Presupuestos y Responsabilidad Hacendaria, que en su artículo 28 señala que el proyecto de Presupuesto de Egresos se presentará y aprobará, cuando menos, conforme a una serie de clasificaciones, entre las cuales se encuentra la de género, que "agrupa las previsiones de gasto con base en su destino por género, diferenciando entre mujeres y hombres".

3. El Decreto de Presupuesto de Egresos de la Federación para el Ejercicio Fiscal de cada año, que incluye un capítulo relativo a la igualdad de género y un anexo específico en que se detallan los recursos del GEMIG. Este anexo, ...., se denominó en un principio "Presupuesto para mujeres y la igualdad de género" y luego pasó a llamarse "Erogaciones para la igualdad entre mujeres y hombres". En él se presenta el presupuesto desagregado por ramos administrativos y programas presupuestarios en los que la Cámara de Diputados etiqueta recursos públicos federales para las mujeres y la igualdad de género.

La igualdad entre mujeres y hombres es un valor fundamental en nuestra Carta Fundamental y una condición esencial para lograr los objetivos de crecimiento y cohesión social. Así, la Constitución Política de los Estados Unidos Mexicanos en su artículo 4o. Señala que : "El varón y la mujer son iguales ante la ley. Esta protegerá la organización y el desarrollo de la familia. ......." Ello ampara que la Ley General para la Igualdad entre Mujeres y Hombres, en su Título I, Capitulo Primero contemple regular y

Público Estatal (Reforma del 26/Dic/2013);Ley de Fiscalización Superior para el Estado de Sonora (Decreto 21/Dic/2015) 
garantizar la igualdad de oportunidades y de trato entre mujeres y hombres, proponer los lineamientos y mecanismos institucionales que orienten a la Nación hacia el cumplimiento de la igualdad sustantiva en los ámbitos público y privado, promoviendo el empoderamiento de las mujeres y la lucha contra toda discriminación basada en el sexo. Sus disposiciones son de orden público e interés social y de observancia general en todo el Territorio Nacional. En lo no previsto en esta Ley, se aplicará en forma supletoria y en lo conducente, las disposiciones de la Ley Federal para Prevenir y Eliminar la Discriminación, la Ley de la Comisión Nacional de los Derechos Humanos, la Ley del Instituto Nacional de las Mujeres, los instrumentos internacionales ratificados por el Estado mexicano y los demás ordenamientos aplicables en la materia.

\section{Consecuencias económicas}

Acorde a los expertos, la equidad es condición sine qua non para la eficiencia económica, si atendemos a la definición de esta última como la optimización de resultados con los recursos existentes en un país. En este sentido, la pobreza infantil, la dificultad de acceso al empleo, a la educación, al deporte, a la vivienda, a la salud o a la protección social ${ }^{32}$, no solamente son inequidades presentes sino que detienen la formación y desarrollo del capital humano de un país. Asimismo, la extrema desigualdad en la distribución de la riqueza y la incipiente democracia son detonantes de serios problemas de violencia, enfermedades, marginación social, deterioro del medio ambiente, formación de grupos de delincuencia entre otros fenómenos que

\footnotetext{
${ }^{32} \mathrm{El}$ empleo informal tiene un peso importante en México más de la mitad de las mujeres mexicanas con un empleo trabajan en el sector informal no agrícola. A través del Programa de Estancias Infantiles para Apoyar a Madres Trabajadoras PEIMT y del Seguro Popular...... México ha realizado avances importantes en la ampliación de servicios y de protección social en salud a los grupos más vulnerables, incluidos los trabajadores informales. El Seguro Popular, además, cuenta con un componente específico para mujeres: la Estrategia de Embarazo Saludable, la cual proporciona atención gratuita antes, durante y después del parto a mujeres afiliadas. Por otro lado, un gran número de trabajadoras informales son trabajadoras domésticas, que por lo general cuentan con condiciones laborales deplorables. Este es un punto a considerar para revertir los efectos negativos con su adecuada regulación, similar a la que debiera realizarse para legislar la protección social que corresponde otorgar el país extranjero que contrate temporalmente a nuestros nacionales, para realizar diversos trabajos en su territorio.
} 
frenan el adecuado funcionamiento social y económico de una región o nación. En el mismo tenor, las externalidades negativas provocadas por las políticas que han intentado potenciar la eficiencia económica descuidando la equidad son gravísimas...... y no solamente exigen un enorme gasto público para revertirlas, sino que pueden llegar a ser irreversibles. ${ }^{33}$

\section{Importancia de la prelación en la solventación de necesidades}

En este contexto, la igualdad de género ocupa un lugar primordial de tal suerte que en la mayoría de los países, se contempla la presencia cada vez mayor de la mujer en el mercado de trabajo y sus logros en la educación y formación son tendencias confortadoras. En México en particular, -los estudios sobre el empleo femenino demuestran que la educación de la mujer, el tiempo de empleo que ocupa, su itinerario laboral, el tipo de pareja que forma, la presencia de hijos pequeños y las ideas que tiene sobe el tipo ideal de familia determinan la permanencia de una mujer en el mercado laboral o en su mayoría pasar a realizar solo tareas domésticas; ${ }^{34}$ en el primer supuesto, en muchos ámbitos laborales persisten importantes disparidades entre hombres y mujeres, en cuanto a una menor retribución por el mismo trabajo, ${ }^{35} \sin$ retribución por los cuidados a personas dependientes o con capacidades diferentes en el hogar y que decir de la casi nula representación en puestos de responsabilidad ${ }^{36}$.

33 Pazos Morán, M., et al., Fiscalidad y Equidad de Género, Primera ed., 2010 Fundación Carolina - CeALCI , Madrid, 2010, pp.1-3

${ }^{34}$ El número de hombres y mujeres que trabajan a tiempo parcial, es un claro indicador de las dificultades persistentes en nuestros días en la conciliación entre la vida familiar y profesional de la mujer y del hombre ya que las mujeres en su mayoría se ven obligadas, con más frecuencia que los hombres, a abandonar el mercado laboral o a recurrir a formas de organización del trabajo que no les permiten explotar plenamente sus capacidades ---autoempleándose en casa en labores de costura,lavado planchado, confección de alimentos entre otros menesteres que no implica salir de casa .

35 INEGI Sistema nacional de Cuentas Nacionales Cuenta Satélite del Sector Salud de México 2008-2011 Salud. Valor del trabajo no remunerado en cuidados de la salud por sexo 2011PPorcentaje :Hombres 27.0,Mujeres 73.0.

36 Las mujeres mexicanas están subrepresentadas en los cargos directivos, ganan menos que los hombres y tienen menores probabilidades que ellos de tener un negocio y emplear a otros: $7 \%$ de los miembros de la junta directiva de las empresas mexicanas son mujeres (10\% en la OCDE) y sólo $2 \%$ de las mexicanas son empresarias (similar al promedio de la OCDE). En cuanto a las brechas de salario, no se cuenta con información para México. 
Estas diferencias también tienen un impacto importante en los ingresos percibidos y en las pensiones de las mujeres. Tener un salario menor significa disponer de una pensión menor, y esto a su vez genera un mayor riesgo de pobreza para las mujeres al llegar a la edad de adultez mayor. Las diferencias salariales entre hombres y mujeres obedecen a un nivel continuo de discriminación y de desigualdades en el mercado laboral que, en la práctica, afectan no solo a las mujeres sino a las familias de las que forman parte, ${ }^{37}$ además, están enlazadas a una serie de factores legales, sociales y económicos cuya importancia se extiende mucho más allá que el solo argumento de "a igual trabajo igual remuneración". En este sentido, la maternidad limita las tasas de empleo femenino, y en el hogar, --como ya se hizo mención-- las mujeres siguen realizando actividades por más horas, sin remuneración, que las que son realizadas por los hombres. Los roles sociales asignados a los sexos siguen influyendo en decisiones individuales trascendentales en educación, salud, organización del trabajo ${ }^{38}$, familia y fertilidad entre otras, las que a su vez, tendrán un impacto en la economía y la sociedad. En contrapartida, tratando de explicitar y hacer realidad la igualdad de género por lo que respecta a las oportunidades en el mercado laboral y al tratamiento en el trabajo en toda la Nación tratando de conciliar la vida profesional y familiar de los hombres trabajadores con hijos, tenemos las reformas aprobadas en México ${ }^{39}$ en relación a las licencias parentales. De acuerdo a la Ley Federal del Trabajo (artículo 132, fracción XXVII Bis),el patrón está obligado a otorgar permiso de paternidad de cinco días laborables con goce de sueldo, a los hombres trabajadores, por el nacimiento de sus hijos y de igual manera en el caso de la

\footnotetext{
${ }^{37}$ Las diferencias de género en México se observan de igual manera en el trabajo no remunerado. En casa, las mujeres mexicanas dedican cuatro horas diarias más que los hombres al trabajo no remunerado. Esto representa el doble de tiempo que la mujer promedio de la OCDE. El trabajo doméstico tiene un efecto negativo sobre el número de horas en el trabajo remunerado de las mujeres y en la brecha salarial de género. Los roles de género constituyen una barrera importante a las oportunidades económicas de México y de las mujeres mexicanas.

${ }^{38}$ Los niveles de empleo de las mujeres, aunque muestran un aumento moderado, son los más bajos de la OCDE después de Turquía (43\% de las mujeres mexicanas tienen un empleo remunerado, comparado con un promedio de la OCDE del 60\%). La modesta participación de las mujeres mexicanas en el mercado laboral se debe en gran parte a la carencia de políticas de conciliación entre trabajo y vida familiar, especialmente la insuficiente oferta de servicios de cuidado infantil (guarderías).

${ }^{39}$ Publicadas en el DOF 30 de noviembre de 2012
} 
adopción de un infante.

Las reformas en materia laboral precitadas se suman a un importante número de ordenamientos jurídicos contra la discriminación por razón del género que tiene nuestro país. No obstante, de todos es sabido que un marco jurídico efectivo requiere supervisión, ejecución, evaluación y actualización continua, además de otras medidas como la formación permanente sobre igualdad de género, en los distintos sectores de la sociedad(universidades, instituciones de educación superior, media superior, secundaria, básica; empresas públicas y privadas, organismos responsables y entidades de la administración pública).

\section{Formulación, diseño y objetivo de la política pública abierta (Metodología)}

Para formular y diseñar en México, una política pública abierta que pretenda solventar o disminuir la inequidad de género, en primer lugar la política pública debe apuntar al compromiso del Estado para hacer cumplir la Ley General para la Igualdad entre Mujeres y Hombres, en segundo lugar promover la participación de hombres y mujeres en el diseño, la elaboración, la aplicación, el seguimiento y la evaluación de los resultados de los programas de la administración pública federal y su consecuente expresión en el presupuesto de egresos de la Federación. ${ }^{40}$.....con esta política se busca asegurar que en el gasto público se destinen recursos a resolver las necesidades de las mujeres derivadas de las desigualdades de género transparencia-- y como un tercer paso que se promueva la creación de condiciones en todos los sectores de la sociedad -- colaboración -- para el logro de la igualdad de género.

Por otra parte en nuestro país .....el etiquetado del gasto para las mujeres y la igualdad de género es una acción afirmativa relevante y una estrategia para incorporar la perspectiva de género en el gasto público e implica trabajar en la asignación presupuestaria adecuada, definir su especificidad y garantizar el seguimiento del presupuesto, con miras a asegurar que los recursos se utilicen en beneficio de las mujeres, tengan un impacto en la desigualdad y contribuyan a acortar las brechas de

40 Comisión Económica para América Latina y el Caribe, CEPAL Políticas ....ibid.,pp.99 
género. ${ }^{41}$ En este sentido, el objetivo anterior se contempla en el artículo 24 del Decreto de Presupuesto de Egresos de la Federación para el Ejercicio Fiscal 2016, donde se incluyen un conjunto de aspectos que se deben considerar para lograr dicho objetivo. El cumplimiento de este propósito lleva implícita la transformación de un presupuesto de egresos tradicional en un presupuesto con perspectiva de género.

Por su parte en el Anexo 13 del Decreto precitado, se detallan de forma agregada las erogaciones de los programas para la igualdad entre hombres y mujeres. Dada la diversidad del gasto etiquetado, hay programas en que se especifica claramente quiénes son las beneficiarias del gasto; en otros casos, las erogaciones se destinan a actividades institucionales, por lo que no se puede identificar a quiénes beneficiarán estos recursos.

Asimismo, los responsables de la implementación de la política son las dependencias y entidades que tienen a su cargo los programas incluidos en el Anexo denominado "Erogaciones para la igualdad entre mujeres y hombres" del Decreto de Presupuesto de Egresos de la Federación para el Ejercicio Fiscal de cada año.

Para asegurar su cumplimiento, el Poder Legislativo y, en particular, la Cámara de Diputados han aprobado disposiciones jurídicas y presupuestarias que incluyen la obligación de informar al Poder Legislativo y a la Secretaría de Hacienda y Crédito Público sobre la manera de ejecutar los recursos etiquetados que permiten hacer viables las políticas públicas para el adelanto de las mujeres y la igualdad de género.

Las dependencias y entidades responsables de los programas incluidos en el anexo mencionado deben informar trimestralmente a través del sistema de información creado por la Secretaría de Hacienda y Crédito Público y del sistema de evaluación de desempeño. Deben detallar, por cada programa, la población objetivo atendida, los indicadores utilizados y la programación y el avance en la ejecución del gasto. Además, tienen la obligación de presentar un informe cualitativo sobre las actividades específicas realizadas y las brechas o desigualdades de género que atendieron, junto con las medidas adoptadas.

Con la metodología anterior para la formulación y el diseño de políticas públicas abiertas para la igualdad de género, en dirección y con el propósito de impulsar un

41 ibid 
avance significativo en los gobiernos de las entidades federativas, ${ }^{42}$ y sus gobiernos municipales resulta ad hoc la formulación de Presupuestos Participativos con Perspectiva de Género, por cuyo medio se podrá apoyar la búsqueda de la equidad y condiciones de empoderamiento económico de las mujeres en sus respectivos territorios, mediante acciones y programas con los que se logre transformar la cultura actual, de manera tal, que: se amplíen sus capacidades y oportunidades de desarrollo, se privilegie aquellas que soporten e impulsen a las mujeres en las acciones que vayan dirigidas hacia las mejoras que garanticen un desarrollo integral familiar y social; que contribuyan al logro de la mujer en su búsqueda de ser activas y protagonistas de sus propio desarrollo, mediante el fortalecimiento de sus capacidades. Asimismo, realizar convenios o acuerdos de apoyo y colaboración con organismos públicos, privados y sociales en función de acciones y negociaciones que favorezcan el logro de mejores condiciones hacia la mujer en los diferentes sectores productivos. Apoyar e impulsar la economía familiar a través de apoyos económicos y formativos. Realizar acciones encaminadas a mejorar la condición de las mujeres y sus familias sobre todo de aquellas que se encuentran en situación de pobreza extrema.

El cumplimiento, por parte del Estado en sentido amplio y restringido, de los compromisos y obligaciones en materia de derechos humanos, depende necesariamente del presupuesto gubernamental, que tiende a considerarse un elemento neutro respecto del género. A través del análisis de la manera en que los presupuestos afectan a las mujeres y a las niñas en comparación con los hombres y los niños, y mediante el etiquetado del gasto, se muestra un marco de análisis crítico que,

42 Excepto el Distrito Federal, Oaxaca, y Jalisco, la mayoría de las entidades federativas y sus municipios incluyendo Sonora aun no cuentan con Presupuestos Participativos con Perspectiva de Género, si bien en forma excepcional municipios como Bacoachi, (desde el 2013 mediante acuerdo de cabildo incorporó de manera transversal en su presupuesto participativo la perspectiva de género), o como el caso del Municipio de Empalme en la presentación del presupuesto por programación del gasto público desde 2013 contempla de manera explícita el rubro de mujeres $\boldsymbol{y}$ el respectivo monto asignado para tal efecto. Asimismo, a partir del 2016 solo 42 municipios mencionan por lo menos una acción a favor de la mujer en forma explicita en sus Planes Municipales de Desarrollo, pero curiosamente en sus respectivos Presupuestos de Egresos solo 29 municipios contemplan el rubro expreso destinado al reglón respectivo, y en contrapartida, los de asistencia social y servicios comunitarios; recreación, deporte y esparcimiento, entre otros rubros, no reflejan ni mucho menos precisan cuanto recurso es destinado a disminuir la brecha existente en las oportunidades que hoy por hoy se ofrece a los hombres y a las mujeres. 
por una parte, permite destinar recursos para las mujeres y las niñas y, por otra, implica una nueva perspectiva acerca de la importancia de asignar recursos presupuestarios destinados a superar la discriminación y crear condiciones que aseguren la igualdad de las personas, sean hombres o mujeres.

Aun cuando todavía queda mucho camino que recorrer, respecto de los objetivos explícitos, el etiquetado representa un avance en el propósito de hacer cumplir mejor la política de igualdad de género, ya que -aún en los municipios que han iniciado con la implementación del presupuesto participativo con perspectiva de género, o mediante el etiquetado de recursos en forma transversal - todavía queda un monto considerable del presupuesto de egresos al que no se ha incorporado la perspectiva de género.

\section{Necesidad de seguimiento}

Si bien la etiquetación del gasto es un avance importante en el camino hacia la igualdad de género y constituye una muestra de la voluntad y el compromiso político existentes en las autoridades municipales, ${ }^{43}$ para institucionalizar e introducir la transversalización de género en la administración pública y en el presupuesto, aún están pendientes la ampliación de la medida, y la creación de instrumentos técnicos y de prácticas continuas apoyadas en la normatividad y otras experiencias nacionales e internacionales exitosas, con miras a incorporar de lleno el enfoque de género en los presupuestos municipales. Por lo que además se hace necesario realizar :

a) La evaluación del impacto que confirme si estos gastos están teniendo el efecto esperado, para en cuyo caso mejorar la política.

b) La revisión de los indicadores y las metas de los programas con GEMIG e identificar su alineación y coherencia con el marco normativo en materia de políticas para la igualdad de género y el acceso de las mujeres a una vida libre de violencia.

c) Observar la repercusión de esta clasificación del gasto y de las políticas a las

${ }^{43}$ Como las de Bacoachi, de Empalme desde 2013 y 27 municipios mas del Estado de Sonora quien hoy por hoy han asignado al efecto, un porcentaje mínimo de sus respectivos presupuestos de egresos. Boletín Oficial Tomo CXC Núm. 53 Sección XXVII 31 de Diciembre de 2012 pp 2 y 6, Boletín Oficial Tomo CXCVII Número 4 Sección III, 14 de enero de 2016 
que se vincula, para evaluar la manera en que el gasto contribuye a cerrar las brechas en materia de igualdad de género.

Además se requiere el compromiso gubernamental para impulsar entre las mujeres la cultura cívica de ciudadanía que contribuya "a una mayor participación en los procesos de política pública abierta y exigir que se respeten sus derechos humanos, con el fin de que la incidencia sea mayor y no quede exclusivamente en manos de las y los expertas (os) y conocedoras (es) de estos temas. " 44

\section{Retos}

Retos a corto (2017-2019) plazo. Planteamos que se cumpla con la Ley de Participación Ciudadana en cuanto al Presupuesto participativo y, vincular dicho instrumento con las prescripciones de la Ley para la Igualdad entre Mujeres y Hombres en el Estado de Sonora LIHYMESON, e incorporen la perspectiva de género. En consecuencia, se deberán implementar los presupuestos participativos de egresos del estado y de los 72 municipios con perspectiva de género.

Aporte :Propuesta de Reformas ${ }^{45}$ a la legislación local, a efecto de que el Estado de Sonora y sus Municipios posean el marco jurídico adecuado para la implementación de los presupuestos participativos con perspectiva de género.

Descripción de la Propuesta:La titular del Poder Ejecutivo y los 72 ayuntamientos deberán revisar los planes estatal y municipales de Desarrollo que rigen sus periodos constitucionales y los proyectos de presupuestos de egresos para 2017, para en su caso realizar las respectivas modificaciones observando la Ley de Participación

44 Ibidem, Políticas públicas para la igualdad de género... 109

\footnotetext{
${ }^{45}$ Presentada el 26 de marzo de 2015,en el Marco del Foro, Gobierno Honesto, Transparente e Innovador, Convocado por la candidata a Gobernadora Claudia Artemisa Pavlovich Arellano por el Partido Revolucionario Institucional, en la Mesa de Trabajo, Sobre Planeación Democrática con el Tema: Presupuestos Participativos con Perspectiva de Género a Nivel Estatal y Municipal. María Inés Aragón Salcido y Adria Velia González Beltrones
} 
Ciudadana en cuanto al Presupuesto participativo y, vincular dicho instrumento con las prescripciones de la Ley de Planeación del Estado de Sonora, a través del Comité de Planeación (COPLADES) y los comités de planeación municipal (COPLAM).

La titular del Ejecutivo del Estado impulsará la igualdad de oportunidades entre mujeres y hombres, a través de la incorporación de la perspectiva de género en el diseño, elaboración y aplicación de los programas de la Administración Pública Estatal. Para tal efecto, las dependencias y entidades deberán realizar lo siguiente en el transcurso de los primeros tres meses de 2017 :

I. Incorporar en los programas la perspectiva de género y reflejarla en sus indicadores y metas;

II. Identificar la población objetivo atendida por los mismos, diferenciada por sexo, grupo de edad, en cobertura estatal o municipal, según corresponda;

III. Fomentar la perspectiva de género en la ejecución de programas que, aun cuando no estén dirigidos a mitigar o solventar desigualdades de género, se puedan identificar de forma diferenciada los beneficios específicos para mujeres y hombres;

IV. Proporcionar la información mencionada en las anteriores fracciones a la Secretaría de Hacienda y al Instituto Sonorense de la Mujer, en el transcurso del primer trimestre de 2017; y

V. Las erogaciones orientadas a la igualdad entre mujeres y hombres, serán materia de un apartado especial en los informes trimestrales y en la cuenta de la hacienda pública estatal del ejercicio fiscal 2017, esto es de manera que se presente la correspondiente relación de actividades institucionales y proyectos de los entes públicos, con los importes asignados y ejercidos, así como los resultados logrados.

Factor de Innovación: Visualizar las brechas de desigualdad en los programas de gobierno, que permitirán promover las acciones afirmativas en los programas sectoriales, regionales, institucionales y especiales.

Viabilidad Técnica y Financiera: Consideramos que es factible estimar el costo del proyecto y su implementaciónen un el plazo estimado del último trimestre del año en 
curso.Se considera que no se presentan hasta el momento obstáculos legales o judiciales, pero se requiere la voluntad política al mas alto nivel para su implementación.

Número estimado y segmento beneficiado con la propuesta: La población femenina del estado 1,322,868 mujeres (mujeres. niñas, adolescentes, adultas y tercera edad).

Compromiso Concreto: Reconocer la desigualdad entre mujeres y hombres en los programas de gobierno y tomar medidas compensatorias.

\section{Bibliografía}

- Comisión Económica para América Latina y el Caribe CEPAL. Benavente María Cristina, Valdés B Alejandra Políticas públicas para la igualdad de género. Un aporte a la autonomía de las mujeres. CEPAL Santiago de Chile,2014

, Trigésimo Sexto período de sesiones, Ed. CEPAL Ciudad de México,23 a 27 de mayo de 2016

- INEGI Sistema nacional de Cuentas Nacionales Cuenta Satélite del Sector Salud de México 2008-2011 Salud.

- Lee, G. and Kwak, Y. H. (2011). An Open Government implementation model: moving to increased public engagement. Washington, DC: IBM Center for the Business of Government.

- Margain Manautou Emilio,Introducción al Estudio del Derecho Tributario, Ed.Porrúa, México, 2014, pp.140

- Organización para la Cooperación y el Desarrollo Económicos OCDE, Serie Mejores Políticas, México Mejores Políticas para un Desarrollo Incluyente, Ed. OCDE, 2012

- Pazos Morán, M., et al., Fiscalidad y Equidad de Género, Primera ed., 2010 Fundación Carolina - CeALCI , Madrid, 2010

- Ramírez-Alujas, Álvaro (2011). "Gobierno abierto y modernización de la gestión pública: tendencias actuales y el (inevitable) camino que se viene". Reflexiones seminales. Revista Enfoques, Vol. IX, no 5, Madrid. 


\section{Webgrafía}

- Boletín Oficial Tomo CXC Núm. 53SeccXXVII 31 de Diciembre de 2012

- Excélsior Periódico Nacional. Demandas magisteriales en voz de la Coordinadora Nacional de Trabajadores de la Educación CNTE, http://www.excelsior.com.mx/nacional/2016/07/27/1107360 ,

- -Expansión datosmacro [online]Disponible en:http://www.datosmacro.com/deficit , Consultado 20/03/2016

- -INFONAVIT Instituto del Fondo Nacional para la Vivienda de los Trabajadores, Plan de Labores y 2016. http://portal.infonavit.org.mx/wps/wcm/connect/5a5a868c-f72e-4efb-be9f167a557ce29a/Plan_de_Labores_y_Financiamientos_2016.pdf?MOD=AJPERE S\&CVID=lb2yf82

- México Evalúa consultado [on line] Disponible en: http://mexicoevalua.org/2014/04/14/descifrando-la-caja-negra-del-gasto/

- SHCP,Secretaría de Hacienda y Crédito Público,[online] Disponible en: http://finanzaspublicas.hacienda.gob.mx/es/Finanzas_Publicas/Informes_al_Con greso_de_la_Union Consultado 20/03/2016

\section{- Legislación}

- Constitución Política de los Estados Unidos Mexicanos CPEUM 2016

- Ley de Fiscalización Superior para el Estado de Sonora (Decreto 21/Dic/2015)

- Ley de Participación Ciudadana del Estado de Sonora

- Ley de Planeación del Estado de Sonora (Reforma del 23/Dic/1991)

- Ley de Presupuesto de Egresos, Contabilidad Gubernamental y Gasto Público Estatal (Reforma del 26/Dic/2013)

- Plan Nacional de Desarrollo 2013-2018

- Programa Nacional de Financiamiento del Desarrollo 2013-18 PRONAFIDE

- Boletín Oficial Tomo CXC Núm. 53SeccXXVII 31 de Diciembre de 2012

- Boletín Oficial Tomo CXCVII Número 4 Sección III, 14 de enero de 2016

- Diario Oficial de la Federación Tercera Sección, Lunes 16 de diciembre de 20 